\title{
Protecting offshore wind turbines against ship impacts by means of adaptive inflatable structures
}

\author{
Cezary Graczykowski* and Jan Holnicki-Szulc \\ Institute of Fundamental Technological Research, SMART- TECH Centre, Polish Academy of Sciences, 21, 00-049 \\ Warsaw, Poland
}

Received 13 September 2007

Revised 2008

\begin{abstract}
Collisions with small service ships are serious danger for offshore wind turbines. Installing torus-shaped adaptive inflatable structure that surrounds a wind turbine tower at water level is one method of effective protection. Proposed pneumatic structure contains several separate air chambers equipped with devices for fast inflation and pressure release. The system can be adapted to various impact scenarios by adjusting the level of initial pressure in each chamber and by controlling the release of compressed air during collision. The paper presents finite element simulation of ship collision with wind turbine tower protected by pneumatic structure, conducted using ABAQUS software. Introduced methods of pressure adjustment are aimed at mitigating tower and ship response. The performed feasibility study proves that inflatable structure can effectively protect the wind turbine tower and the ship against serious damage.
\end{abstract}

Keywords: Adaptive inflatable structures, pneumatic structures, adaptive impact absorption, flow control, offshore structures, ship collisions

\section{Introduction}

Wind turbines are the main source of renewable energy. Moreover, the contribution of wind energy to global energy production is still increasing and it is assumed that wind energy production in EU countries will have grown to $150 \mathrm{GW}$ by 2020 . The largest wind generators currently operating provide up to $3.6 \mathrm{MW}$ power and an increase of their effectiveness is still required. It can be achieved by locating wind turbines in regions where the wind conditions are more beneficial, for instance in offshore regions where the wind flows smoothly and briskly. Additional advantages of locating wind turbines offshore include the availability of large open spaces and the lack of noise and aesthetics-related inconveniences for inhabitants. Wind turbines are usually situated in shallow continental shelves in the vicinity of large ports (for example on Northern Sea). Such locations incur a comparatively low cost for wind turbine installation and energy transportation.

In offshore regions wind turbines are exposed to harsher environmental conditions. The main threats for offshore wind generators are very strong winds and ice loading in winter, cf. [1]. Typical method of reducing ice forces acting on a structure is using ice braking cones which serve as passive or semi-active tuned mass dampers as proposed by Kärnä, cf. [2] and Mróz, cf. [3]. Another substantial problem is the risk of large tanker vessel collision and the consequent serious environmental pollution. The indications for the design of offshore wind turbines which reduces

\footnotetext{
${ }^{*}$ Corresponding author: Institute of Fundamental Technological Research, SMART- TECH Centre, Polish Academy of Sciences, $21,00-049$ Warsaw, Poland. E-mail: cgraczyk@ippt.gov.pl; web page: http://smart.ippt.gov.pl.
} 

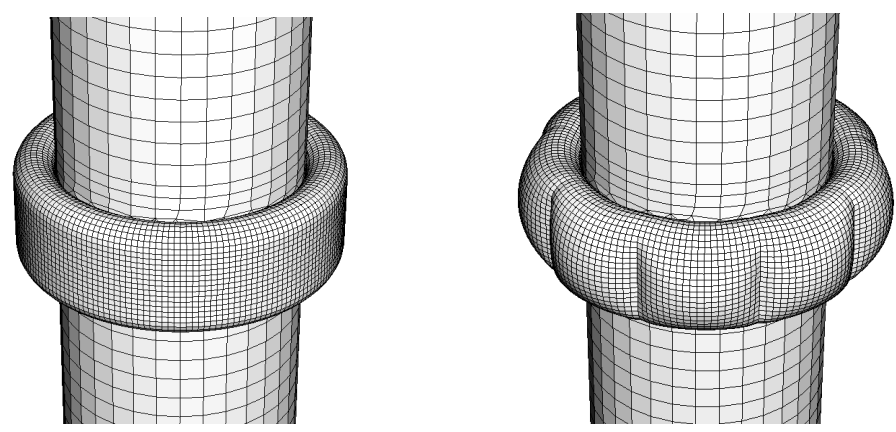

Fig. 1. Adaptive Inflatable Structure surrounding the wind turbine tower.

the possibility of the tanker vessel damage were given by Lehmann et all [4] and Biehl [5]. Additional dangers for offshore structures are collisions involving small service ships which have to dock to wind turbine towers for the purpose of maintenance and monitoring. Such collisions occur especially often during rough sea conditions and can lead to serious damage to both the wind generator tower and the ship. Therefore, an additional structure providing safety for docking operations is required. In this paper, an idea of Adaptive Inflatable Structure (AIS) attached to the wind turbine tower is proposed and its feasibility verified.

The introduced solution belongs to so called Adaptive Impact Absorption (AIA) approaches. AIA focuses on active adaptation of energy absorbing structures to extreme overloading by means of system of sensors detecting and identifying impact in advance and controllable dissipaters, so called structural fuses, cf. [6,7]. A semi-active or fully active solutions are usually realised via dissipative devices (with no need for significant power supply) based on magneto-rheological fluids or piezo actuated valves, cf. [8]. The novelty of presented inflatable structure relies upon controlling release of compressed air as an efficient method of impact absorption.

The paper is organised in the following way: initially, the background of AIS is introduced and key dynamic equations are developed. Then, full model of the wind turbine is presented and reduced to the water level. Three models of ship collision with the tower protected by inflatable structure are considered. The most precise, three dimensional model is used to compute the response of the passive structure and it verifies the correctness of simpler models. Two dimensional, computationally lighter model allows to apply pressure regulation methods oriented toward mitigation of ship and tower response. The simplest, one dimensional model can be used to estimate the response of the structure without conducting finite element analysis. The main goal of the paper is to compare the results obtained by applying various pressure adjustment schemes and to prove the superiority of AIA approach (i.e. semi-active and active solutions) in comparison to baseline passive case.

\section{Idea of adaptive inflatable structure}

The Adaptive Inflatable Structure (AIS) that will be used for protecting offshore wind turbine against collisions of small ships is torus-shaped and surrounds the tower, see Fig. 1. The AIS is located at the water level and it may be partially submerged. The dimensions of the inflatable structure are limited to two meters in height and around one meter in width due to requirements of fast inflation and pressure release during impact. The walls of the pneumatic structure can be made of rubber reinforced by steel rods or any other material which provides high durability and allows large deformations during ship impact. To achieve better adaptation to various impact scenarios, the inflatable structure is divided into several separate air chambers located around the tower, as shown in Figs 1 and 2 . The exact design of the AIS is determined by the range of possible impact energies and it will be precisely defined while considering the conditions for optimal impact absorption.

The inflatable structure should be permanently inflated to a relatively low pressure which provides mitigation of weaker impacts. Additional inflation is planned before any stronger collision. It is executed for each chamber separately by a compressor located inside the tower or alternatively by a fast-reacting pyrotechnic system acting similarly to the one in a car air bag. The gas pressure acting outwards increases global stiffness of the torus and repels the ship outside the tower. This way the inflatable structure prevents direct collision between the ship and 


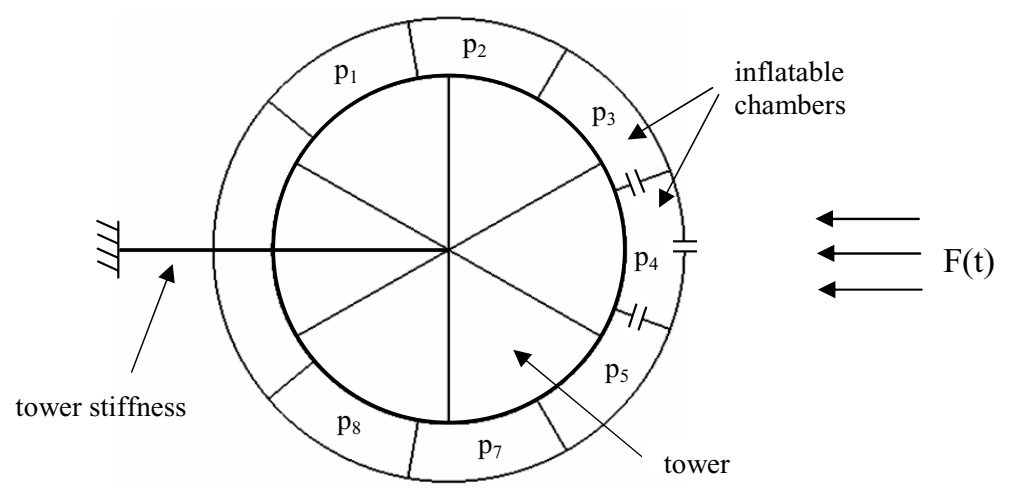

Fig. 2. Horizontal intersection of the inflatable structure for protection of a tower.

the tower and potential excessive forces and accelerations. Utilization of compressed air causes that considered protective structure can be easily adapted to various impact forces and scenarios. Value of initial internal pressure can be adjusted and varied between the chambers according to hitting object velocity, its mass and the area of contact with pneumatic structure.

During the collision with an external object controlled release of pressure is executed by opening controllable high performance valves. Such valves are mounted in external walls of inflatable structure and in diaphragms between pressurised packages so they allow the gas flow between chambers and outside the structure. Release of pressure allows to control stiffness of the pneumatic structure in the subsequent stages of impact. This way the impacting object can be stopped using the whole admissible stroke and its acceleration can be significantly reduced. Additionally, controlled release of compressed air efficiently dissipates the impact energy.

The adaptation procedure can be successfully applied assuming that the ship impact is sufficiently well recognized. The initial ship velocity and impact direction can be measured by means of ultrasonic velocity sensors, cf. [9]. Moreover the system can be equipped with accelerometers, piezoelectric pressure sensors and LVDT displacement sensors located inside the torus chambers, which help to follow the actual dynamic response of the system. Impacting object mass and its initial kinetic energy can be recognized during the initial stage of impact by using results from above sensors and by applying procedures described in detail in [10].

The purpose of applying pneumatic structures is to mitigate the response of both the ship and the wind turbine tower. In particular, the inflatable structure helps to minimize ship deceleration, avoid ship rebound, decrease stresses arising at the location of the collision and mitigate tower vibrations.

\section{Equations describing inflatable structures}

Numerical analysis of the pneumatic structure subjected to an impact load requires considering the interaction between its walls and the fluid enclosed inside. An applied external load causes large deformation of the structure and change of the capacity and pressure of the fluid. The pressure exerted by the fluid affects, in turn, the deformation of the structure and its internal forces.

The most precise method for solving fluid-structure interaction problems is so called Arbitrary Lagrangian Eulerian (ALE) approach where Navier-Stokes equations for the fluid are solved in Euler reference frame and structural mechanics equations in Lagrange reference frame. Such approach is frequently applied for extremely fast processes like airbag deployment (cf. [11]) or when the recognition of the flow pattern is the main problem addressed. In the problem considered, fluid part of the analysis is used to compute global forces acting on the walls of inflatable structure and the exact space variation of fluid pressure and velocity is not of interest. Moreover, the impacting object velocity is much lower than the speed of impulse propagation in gas so pressure becomes constant across the chambers relatively fast. Therefore, we will use so called Uniform Pressure Method (UPM) which assumes that gas is uniformly distributed inside each chamber and chamber walls are subjected to uniform pressure. 
The dynamics of the inflatable structure is described by the nonlinear equation of motion, whose general form reads:

$$
\begin{aligned}
\mathbf{M} \ddot{\mathbf{q}}+\mathbf{C \dot { q }}+\mathbf{K}(\mathbf{q}) \mathbf{q} & =\mathbf{F}(\mathbf{p}, \mathbf{q})+\mathbf{F}_{I} \\
\mathbf{q}(0) & =\mathbf{q}_{0}, \dot{\mathbf{q}}(0)=\mathbf{V}_{0}
\end{aligned}
$$

where matrices $\mathbf{M}, \mathbf{C}, \mathbf{K}$ indicate mass, damping and stiffness and $\mathbf{q}$ denotes vector of degrees of freedom. The initial conditions are imposed on vector $\mathbf{q}$ and its first time derivative $\dot{\mathbf{q}}$ by assuming initial displacements $\mathbf{q}_{0}$ and velocities $\mathbf{V}_{0}$. Vector $\mathbf{p}=\left\{p_{1}(t), p_{2}(t), \ldots, p_{n}(t)\right\}$ indicates gauge pressures in the cavities. The impact can be modelled by r.h.s. force vector $\mathbf{F}_{I}$, by initial conditions or by contact defined between the inflatable structure and other object. In any case, the $\mathbf{F}(\mathbf{p}, \mathbf{q})$ vector is present in the problem, since it provides the coupling with the internal fluid. The interaction of the structure and the fluid during large deformation can be correctly taken into account only by assembling the equilibrium equations in actual configuration so equation of motion has to be considered in a nonlinear form.

We assume that each cavity of the inflatable structure is filled with a compressible (pneumatic) fluid, which is described analytically by the equation of state for ideal gas:

$$
\begin{aligned}
& \bar{p}(t)=\rho(t) R \bar{T}(t) \\
& \text { or } \bar{p}(t) V(t)=m(t) R\left(T(t)-T_{Z}\right)
\end{aligned}
$$

Absolute pressure $\bar{p}$ is defined as $\bar{p}=p+p_{A}$ where $p$ is gauge pressure and $p_{A}$ is an ambient pressure, the absolute temperature is defined as $\bar{T}=T-T_{Z}$ where $T$ is current temperature on the Celsius scale and $T_{Z}$ is absolute zero temperature. The gas constant $R$ is related to the universal gas constant $\bar{R}$ and molecular weight MW by the formula: $R=\bar{R} / M W$. Moreover, the variables $\rho, V, m$ indicate gas density, volume and mass, respectively. The initial conditions for the fluid are given by: $\bar{p}(0)=p_{0}, T(0)=T_{0}$. In case when fluid flow occurs, change of fluid mass in the cavity is described by the equation:

$$
m(t)=m_{0}+\int_{0}^{t} q(\bar{t}) d \bar{t}=m_{0}+\int_{0}^{t} q_{\text {in }}(\bar{t})-q_{\text {out }}(\bar{t}) d \bar{t}
$$

where $q_{i n}$ is the mass flow rate into the cavity and $q_{\text {out }}$ is the mass flow rate outside the cavity. The direction of the flow depends on the sign of pressure difference between the cavities. In the simplest considered model of the flow the mass flow rate is related to pressure difference according to the formula:

$$
\Delta p(t)=C_{V} q(t)+C_{H} q(t)|q(t)|
$$

where $\Delta p(t)=p_{\text {out }}-p(t), C_{V}$ is the viscous resistance coefficient, and $C_{H}$ is the hydrodynamic resistance coefficient. Both these coefficients depend on the area and shape of the orifice and they can be found experimentally for given type of the valve. Under the assumption of an isothermal process (or arbitrarily given change of temperature) a set of Eqs (1-4a) fully describes the coupling between the fluid and the surrounding structure. Alternatively, flow of the gas through the orifice can be described by the model which assumes that the flow is blocked at critical velocity. In such case mass flow rate is defined by Saint-Venant formula, cf. [12]:

$$
|q(t)|=C A \frac{\overline{p_{e}}}{\sqrt{R\left(T-T_{Z}\right)}} \sqrt{\frac{2 \chi}{\chi-1}\left(\left(\frac{\bar{p}_{o}}{\bar{p}_{e}}\right)^{\frac{2}{\chi}}-\left(\frac{\bar{p}_{o}}{\bar{p}_{e}}\right)^{\frac{\chi+1}{\chi}}\right)}
$$

where $C$ is the discharge coefficient, $A$ is the orifice area, $\bar{p}_{e}$ is the absolute pressure in the upstream fluid cavity, $\bar{p}_{0}$ is the absolute pressure in the orifice given by:

$$
\begin{array}{lll}
\bar{p}_{o}=\bar{p}_{a} & \text { if } & \bar{p}_{a} \geqslant \bar{p}_{c} \\
\bar{p}_{o}=\bar{p}_{c} & \text { if } & \bar{p}_{a}<\bar{p}_{c}
\end{array}
$$

where $\bar{p}_{a}$ is equal to the ambient pressure or the downstream cavity pressure. The critical pressure $\bar{p}_{c}$ at which chocked or sonic flow occurs is defined as: 


$$
\bar{p}_{c}=\bar{p}_{e}\left(\frac{2}{\chi+1}\right)^{\frac{\chi}{\chi-1}} \quad ; \quad \chi=\frac{c_{p}}{c_{V}}
$$

where $\chi$ is an adiabatic exponent defined as ratio of constant pressure heat capacity $c_{p}$ and constant volume heat capacity $c_{V}$ and for the air $\chi=1.41$.

In general case temperature of the gas can be treated as subsequent unknown of the problem. The balance of the heat transferred to the system $d Q$, enthalpy of the gas added to (removed from) the system $d m_{\text {in }} \bar{H}_{\text {in }}\left(d m_{\text {out }} \bar{H}_{\text {out }}\right)$, change of gas internal energy $d(m \bar{U})$ and the work done by gas $d W$ is given by the first law of thermodynamics for an open system:

$$
d Q+d m_{\text {in }} \bar{H}_{\text {in }}-d m_{\text {out }} \bar{H}_{\text {out }}=d(m \bar{U})+d W
$$

Specific gas enthalpy and specific gas energy are defined as:

$$
\bar{H}_{i n}(t)=c_{p} \bar{T}_{i n}(t) ; \quad \bar{H}_{\text {out }}(t)=c_{p} \bar{T}(t) ; \quad \bar{U}(t)=c_{V} \bar{T}(t)
$$

The flow of the heat across the cavity walls is described by equation:

$$
\frac{d Q(t)}{d t}=\lambda A(t)\left(T_{e x t}-T(t)\right)
$$

where $\lambda$ is the heat conductivity coefficient of the cavity wall and $A(t)$ is the total area of the cavity walls. Finally, work done by gas is defined as:

$$
d W(t)=\bar{p}(t) d V(t)
$$

If the wall of the cavity is perfect insulator $(\lambda=0)$ or when the process is very fast, adiabatic conditions are fulfilled and no heat transfer through the chamber walls occurs: $d Q=0$. In case when the gas flows into the considered chamber Eq. (5a) reads:

$$
\frac{\dot{T}(t)}{\bar{T}(t)}=\left(\chi \frac{\bar{T}_{i n}(t)}{\bar{T}(t)}-1\right) \frac{\dot{m}(t)}{m(t)}-(\chi-1) \frac{\dot{V}(t)}{V(t)}
$$

In case when gas flows out of the chamber Eq. (5a) can be solved analytically:

$$
\frac{\bar{T}(t)}{\bar{T}_{0}}=\left(\frac{m_{0}}{V_{0}} \cdot \frac{V(t)}{m(t)}\right)^{1-\chi} \text { or } \frac{\bar{p}(t)}{\bar{p}_{0}}=\left(\frac{m_{0}}{V_{0}} \cdot \frac{V(t)}{m(t)}\right)^{-\chi}
$$

Finally, when no flow of the gas occurs (i.e. mass of the gas in the cavity remains constant) we obtain well known equation:

$$
\bar{p}(t) \cdot V(t)^{\chi}=\bar{p}_{0} \cdot V_{0}^{\chi}=\text { const. }
$$

\section{Model of the wind turbine tower}

Let us analyse the finite-element model of a typical wind turbine, as shown in Fig. 3, which was introduced in [3]. Considered tower is $88 \mathrm{~m}$ high and its foundation is located $6 \mathrm{~m}$ below the water level. The tower consists of beam elements with circular sections and an external radius varying from 1.16 to $2.11 \mathrm{~m}$. The wind turbine has three 40 meters long blades which are modelled by shell elements. Four flanges located along the tower and three components of the turbine are modelled by point masses. Moreover, the tower is equipped with the cone structure located at the water level which serves as a protection against ice loading. In present case this cone will be replaced by inflatable structure destinated to mitigate collisions of service ships.

Part of the tower located nearby the water level is of greatest interest because of the collision considered. Reduction to one dimensional model at the water level can be performed by means of modal analysis, cf. [13]. For every particular vibration mode, the mass, stiffness and damping of the reduced model are given by the formulae:

$$
M=\frac{1}{\phi_{n C}} \int_{0}^{H} m(z) \phi_{n}^{2}(z) d z=\frac{M_{n}}{\phi_{n C}^{2}}
$$


Table 1

Parameters of the 1D model at the water level

\begin{tabular}{cccrccc}
\hline & $\mathrm{f}_{n}[1 / \mathrm{s}]$ & $\phi_{n C}$ & $\mathrm{M}_{n}[\mathrm{~kg}]$ & $\mathrm{M}[\mathrm{kg}]$ & $\mathrm{K}[\mathrm{N} / \mathrm{m}]$ & $\mathrm{C}[\mathrm{Ns} / \mathrm{m}]$ \\
\hline Mode 1 & 0.33147 & $1.584 \mathrm{e}-3$ & 116748 & $0.4653 \mathrm{e} 11$ & $0.2018 \mathrm{e} 12$ & $0.9690 \mathrm{e} 9$ \\
Mode 2 & 0.38503 & $1.021 \mathrm{e}-3$ & 34646 & $0.3318 \mathrm{e} 11$ & $0.1942 \mathrm{e} 12$ & $0.8029 \mathrm{e} 9$ \\
\hline
\end{tabular}

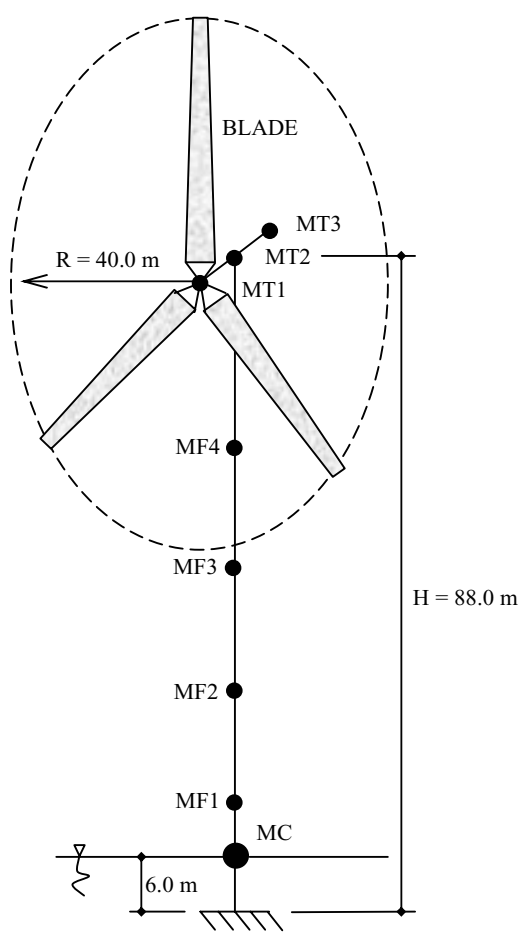

Blades (shell elements):

first own frequency $\omega_{1}=1.30 \mathrm{~Hz}$ weight of blade $\mathrm{G}_{\mathrm{B}}=7006 \mathrm{~kg}$

Tower (beam elements):

47 elements with circular section

radius $\mathrm{R}=1.16 \div 2.11 \mathrm{~m}$

thickness $\mathrm{t}=0.014 \div 0.040 \mathrm{~m}$

weight of tower $\mathrm{G}_{\mathrm{T}}=186782 \mathrm{~kg}$

Point masses (MASS elements): MT1 $=13000 \mathrm{~kg}$

$\mathrm{MT} 2=31800 \mathrm{~kg} \quad$ turbine

MT3 $=35000 \mathrm{~kg}$

$\mathrm{MF} 1=4200 \mathrm{~kg}$

MF2 $=3200 \mathrm{~kg} \quad$ flanges

$\mathrm{MF} 3=1900 \mathrm{~kg}$

MF4 $=2300 \mathrm{~kg}$

$\mathrm{MC}=49620 \mathrm{~kg}$ - ice loading

protecting cone

Total weight of the structure:

$\mathrm{G}=3 \times 7006 \mathrm{~kg}+186782 \mathrm{~kg}+141020 \mathrm{~kg}=$ $=348820 \mathrm{~kg}$

Fig. 3. Numerical model of the wind turbine tower by A. Mróz [3].

$$
K=\omega_{n}^{2} M=\left(2 \pi f_{n}\right)^{2} M
$$

$$
C=2 \xi \omega_{n} M
$$

where: $\phi_{n}(z)$ is the mode shape, $\phi_{n C}$ is the normalized value of the mode on the water level, $M_{n}$ is the generalized mass calculated for this mode and $\omega_{n}$ is the circular frequency. The values of $\phi_{n C}, M_{n}, f_{n}$ can be obtained directly from modal analysis of the structure in Fig. 3. The damping coefficient $\xi$ is assumed to be equal $0.5 \%$. The resulting mass, stiffness and damping parameters of the reduced 1D model are presented in Table 1 and they will be effectively exploited in further sections.

\section{Numerical modelling of collision between ship and tower}

\subsection{Three dimensional model}

For the purpose of precise modelling of the inflatable structure influence on the ship impact against the offshore wind turbine tower, a three-dimensional finite element model was implemented, as shown in Figs 1 and 4 . The model contains only lower part of the wind turbine tower and upper part is modelled by additional masses and forces applied at the upper edge. The tower consists of shell elements with the thickness increased on the water level and 



Fig. 4. Three dimensional model of collision: initial inflation and deformation during collision.
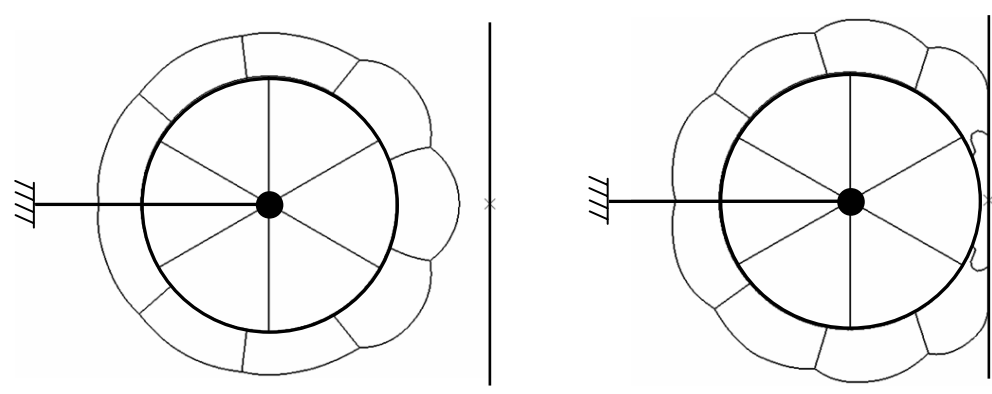

Fig. 5. Two dimensional model of collision: initial state and resulting deformation.

the torus-shaped inflatable structure consists of membrane elements. The gas inflating the chambers is modelled by using 'surface-based cavities' available in ABAQUS software, cf. [14] and operating according to formulae given in Section 3. In considered case the major part of pneumatic torus is located above the water and only a small part is submerged so pressure exerted by water is not taken into account. Typical deformation of the torus caused by internal gas pressure is presented in Fig. 4 a.

Impact of the ship is defined as a contact problem. The ship is modelled as a rigid surface with a prescribed mass and area, approaching the tower with an initial velocity, see Fig. 4. The contact conditions are defined between the ship and the rubber wall of inflatable structure and between the rubber wall and the wall of the tower. When the collision occurs AIS walls deform, chamber volume decreases (cf. Fig. 4b) and pressure rises. During this stage the release of the gas is controlled by changing the flow resistance coefficients. Finally, the ship is stopped and possibly bounces from the inflatable structure. In case when impact energy is too high or control is adjusted improperly, the pneumatic structure is not able to stop the ship and collision against the tower wall occurs.

The main numerical tool used for 3D simulations was the finite element code ABAQUS/Explicit, cf. [14] which uses an explicit scheme of solution and hence is well suited for fast dynamics or strongly nonlinear problems, cf. [15]. For performing optimal pressure release the connection to MATLAB software was established and its optimization procedures were utilized.

\subsection{Two dimensional model at the water level}

Two-dimensional model related to water level was implemented to reduce the time of analysis and to examine various options for inflatable structure design. The model consists of elastic Timoshenko beam elements applied for the tower and AIS walls. The additional mass obtained from a reduction of the full model (according to Table 1) is located in the middle of the structure. Thus, additional elements connecting the mass with the walls of the tower are required, cf. Fig. 5. The stiffness of the tower is modelled by an additional element connected to its middle point.

The numerical software used for the analysis of 2D model was ABAQUS/Standard which solves nonlinear equilibrium equations by implicit method, cf. [16]. The strong advantage of this solver is the possibility of adding 


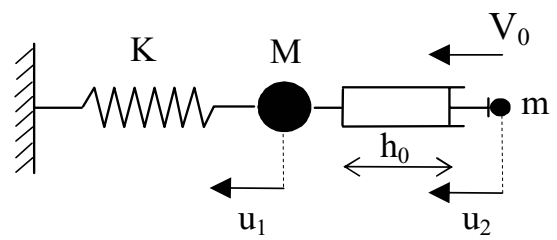

Fig. 6. Two degree of freedom model of collision between the ship and the tower.

FORTRAN subroutines to implement user-defined capabilities. Such subroutines were added to define an adiabatic process, Saint-Venant model of the gas flow and to control release of compressed air (cf. Section 7).

A parametric analysis performed on a two-dimensional model was used to investigate basic features of the inflatable structure. Various dimensions, number of chambers, Young modulus and thickness of the AIS wall were considered. Indications for a proper choice of these parameters are as follows:

- value of pressure required to stop the ship before hitting the tower wall can be decreased by using wide chambers (i.e. torus structure with large external radius)

- wide chambers are more beneficial for reduction of ship acceleration

- too long (along the tower perimeter) chambers can not absorb strong impacts with initial atmospheric pressure and additional inflation is required

- using longer chambers helps to decrease the local stresses in the tower wall

- in long and narrow chambers large deformations and stresses appear after the initial inflating in the outer wall

- using more chambers allows us to adjust pressure more precisely to the actual loading conditions

Taking into account all above mentioned conditions, the maximal admissible pressure, allowable stress of rubber and the maximal initial increase of chamber volume it was decided to divide the inflatable structure into 9 chambers of a width $0.7 \mathrm{~m}$ and construct a $1 \mathrm{~cm}$ wall made of reinforced rubber of vicarious Young modulus equal $150 \mathrm{MPa}$.

\subsection{Simplified 1d spring-piston model}

Finally, ship collision with a tower protected by a pneumatic structure was reduced to two degree of freedom system with single air chamber modelled by an air spring (see Fig. 6), which provides a basic estimation of required pressure values and assessment of the AIS efficiency.

The overall force coming from the compressed air equals $A\left(\bar{p}(t)-p_{A}\right)$ and it acts when the ship is in contact with the inflatable structure. The fact that pneumatic force always repels the tower and the ship is taken into account by neglecting the term $A\left(\bar{p}(t)-p_{A}\right)$ when it becomes negative. Moreover, the pneumatic force disappears after the ship rebound when the chamber exceeds its original size. The response of the system can be divided into two stages: the first one indicating impact and the second one when mass $\mathrm{M}$ is performing free vibrations.

The equations governing the problem read:

$$
\begin{aligned}
& M \frac{d^{2} u_{1}(t)}{d t^{2}}+K u_{1}(t)-\left\{\begin{array}{ll}
\left(\bar{p}(t)-p_{A}\right) A & \begin{array}{c}
\text { for } \bar{p}(t) \geqslant p_{A} \text { and } \\
\text { otherwise }
\end{array} \\
0 & u_{2}(t) \geqslant u_{1}(t)
\end{array}\right\}=0 \\
& m \frac{d^{2} u_{2}(t)}{d t^{2}}+\left\{\begin{array}{lll}
\left(\bar{p}(t)-p_{A}\right) A & \text { for } \bar{p}(t) \geqslant p_{A} \text { and } u_{2}(t) \geqslant u_{1}(t) \\
0 & \text { otherwise }
\end{array}\right\}=0 \\
& I C: \dot{u}_{2}(0)=V_{0}
\end{aligned}
$$

For the impact stage the Eqs (2-5) hold and moreover capacity of the air chamber is defined as:

$$
V(t)=A\left(h_{0}+u_{1}(t)-u_{2}(t)\right)
$$

Summing up the Eq. (8a) integrated over $u_{1}$ and Eq. (8b) integrated over $u_{2}$ gives the balance of the energy for the whole system:

$$
\frac{1}{2} M V_{1}(t)^{2}+\frac{1}{2} K u_{1}(t)^{2}-\int_{0}^{u_{1}-u_{2}}\left(\bar{p}(t)-p_{A}\right) A d\left(u_{1}-u_{2}\right)+\frac{1}{2} m\left[V_{2}(t)^{2}-V_{0}^{2}\right]=0
$$


By using definitions of kinetic energy, potential energy and work done by gas we obtain the formula:

$$
-\Delta E_{k}^{\text {ship }}+W^{\text {atm }}=E_{k}^{\text {tower }}+E_{p}^{\text {tower }}+\Delta U-\Delta Q-\Delta H
$$

which clearly indicates that change of the ship kinetic energy $\left(\Delta E_{k}^{s h i p}\right)$ and work done by atmospheric pressure $\left(W^{a t m}\right)$ are converted into kinetic and potential energy of the tower $\left(E_{k}^{t o w e r}, E_{p}^{\text {tower }}\right)$, internal gas energy, heat transferred to environment and enthalpy of the gas removed from the system.

Since the tower displacement is very small in comparison to the ship displacement we can neglect the term $u_{1}(t)$ in the definition of the air-spring volume Eq. (9). In such case the Eq. (8) describing the system are separated. The second, nonlinear equation can be solved in order to find the ship displacement and the solution of the first equation can be derived by treating its last term as time-dependent excitation. In case when the mass of the gas inside cylinder remains constant, energy balance at the moment when the ship stops is described by the equations:

$$
\begin{aligned}
& \frac{1}{2} m V_{0}^{2}+p_{A} A u_{2}^{\max }=\bar{p}_{0} h_{0} A \ln \left(\frac{h_{0}}{h^{\min }}\right) \\
& \frac{1}{2} m V_{0}^{2}+p_{A} A u_{2}^{\max }=\frac{\bar{p}_{0} h_{0} A}{\chi-1}\left[\left(\frac{h_{0}}{h^{\min }}\right)^{\chi-1}-1\right]
\end{aligned}
$$

for the isothermic and adiabatic process, respectively. In above formulae $u_{2}^{\max }$ indicates maximal ship displacement and $h^{\mathrm{min}}$ indicates minimal cylinder length.

The problems of pressure minimization and ship acceleration minimization are for considered spring-piston model equivalent. The optimal acceleration which prevents collision of both masses is constant in time and can be derived from the kinematics of the structure. The corresponding optimal pressure inside the cylinder can be calculated from the energy balance. We obtain formulae for minimal acceleration and pressure:

$$
\ddot{u}_{2}^{o p t}=-V_{0}^{2} / 2 h_{0}, \quad \bar{p}^{o p t}=\frac{m V_{0}^{2}}{2 h_{0} A}+p_{A},
$$

which hold until ship displacement equals $h_{0}$ and its velocity is reduced to zero. At this moment no gas remains in the chamber and there is no force pushing the ship away from the tower. Hence, ship acceleration is reduced to zero and ship rebound is completely mitigated. Applied pressure release strategy causes that ship impact can be treated as inelastic collision, whereas it resembles elastic collision in passive case. Due to the fact that impulse transmitted to the tower in inelastic collision is two times smaller, initial velocity of the tower after impact is reduced twice.

Corresponding optimal cylinder volume can be calculated from geometrical relations and optimal mass of the gas from ideal gas law. Let us assume the mass flow rate definition according to Eq. (4a) with coefficient $C_{H}$ equal to zero. Formula defining time evolution of optimal viscous flow resistance coefficient in terms of impacting mass, and impact velocity reads:

$$
C_{V}(t)=\frac{\left(\bar{p}^{o p t}-p_{A}\right) R T_{0}}{\bar{p}^{o p t} A \dot{u}_{2}^{o p t}(t)}
$$

Above solution is valid for both isothermic and adiabatic process and it holds when no restrictions on flow resistance coefficient are imposed.

\subsection{Comparison of different collision models}

Results obtained by using three above models (full 3D, 2D at the water level and 1D spring-piston model) were compared with each other in order to verify correctness and limitations of simplified modelling. Comparison of the basic dynamic response of passive structure (pressure and volume of the front chamber, ship acceleration and stress in the tower wall) computed for two impact scenarios of 60 ton ship is presented in Table 2.

Initially it was found that the global stiffness of the chambers in 2D model is too small since the connection of each chamber with the tower wall at the top and bottom of inflatable structure is not taken into account. Thus, radial deformation of 2D structure due to strong initial inflation is larger than the deformation of 3D structure. Moreover, 2D modelling does not capture the vertical deformation of the chambers so ship-torus contact area and front chamber 
Table 2

Comparison of results obtained by using different models of collision

\begin{tabular}{cccccccc}
\hline & $\begin{array}{c}\text { Velocity } \\
{[\mathrm{m} / \mathrm{s}]}\end{array}$ & $\begin{array}{c}\overline{\mathbf{p}}_{0} \\
{[\mathrm{~atm}]}\end{array}$ & $\begin{array}{c}V_{0} \\
{\left[\mathrm{~m}^{3}\right]}\end{array}$ & $\begin{array}{c}\overline{\mathbf{p}}^{\text {max }} \\
{[\mathrm{atm}]}\end{array}$ & $\begin{array}{c}V^{\text {min }} \\
{\left[\mathrm{m}^{3}\right]}\end{array}$ & $\begin{array}{c}\text { Accel. } \\
{\left[\mathrm{m} / \mathrm{s}^{2}\right]}\end{array}$ & $\begin{array}{c}\text { Stress } \\
{[\mathrm{MPa}]}\end{array}$ \\
\hline 1D & 3 & 2 & 2.91 & 4.11 & 1.41 & 17.8 & - \\
2D & 3 & 2 & 2.95 & 3.74 & 1.54 & 19.5 & 84.2 \\
3D & 3 & 2 & 2.60 & 3.52 & 1.47 & 21.8 & 33.8 \\
1D & 6 & 4 & 3.26 & 10.68 & 1.22 & 58.6 & - \\
2D & 6 & 4 & 3.57 & 8.92 & 1.55 & 58.4 & 243 \\
3D & 6 & 4 & 2.97 & 9.72 & 1.22 & 68.9 & 108 \\
\hline
\end{tabular}

volume computed for the same ship position (i.e. distance from the tower) are different for both models. As a result, maximal pressure and acceleration obtained from two and three dimensional model are not precisely equal.

Conducted simulations indicate that maximal pressure obtained by using 2D model is underestimated for fast impacts and overestimated for slow impacts. Accelerations of the ship computed for $2 \mathrm{D}$ case are always lower than the accelerations for full 3D model. Maximal Mises stress in the tower wall obtained by using both models can not be directly compared due to different geometry of the wall, however it was found that stress computed for two dimensional model is usually around 2.5 times larger. General conclusion from above comparison states that two dimensional modelling captures the dynamic response the most appropriately when inflatable structure becomes long and narrow.

Simplified 1D modelling completely neglects initial expansion of the air chambers (expansion coefficient is assumed arbitrary) and its transverse deformation. Obviously 1D model can not be used to compute stresses in tower wall. Simplified spring-piston modelling can be used for rough estimation of basic dynamic response.

\section{General guidelines for pressure adjustment}

The main task of the inflatable torus is to prevent direct impact of the ship against the tower wall. When the inflatable structure is not sealed and inflated it can stop the object of comparatively low kinetic energy of $0.2 \mathrm{MJ}$. In case of stronger impacts inflation of the cavities is required. Due to the fact that the front cavity (the most exposed to impact) is crucial for overall properties of inflatable structure, it is inflated with the highest pressure. Chambers adjacent to the front chamber are auxiliary and their pressure is usually set to $30-50 \%$ of pressure in the front cavity.

Let initially consider the system where only initial pressure is adjusted and all valves remain closed. Value of initial pressure which provides that the ship is stopped in the very vicinity of the tower wall will be referred to as minimal initial pressure $p_{0}^{\min }$. Value of this pressure was calculated for various impact energies using two-dimensional finite element model, cf. Table 3. The assumption of isothermic process was applied. The first example shows the impact of energy $0.756 \mathrm{MJ}$ ( $29.5 \%$ of maximal energy considered) can be absorbed by using initial atmospheric pressure in the front chamber. Maximal pressure $\bar{p}^{\max }$ corresponds to minimal volume of the chamber obtained when the ship nearly touches the tower wall. Maximal front chamber pressure, highest stress arising in the tower wall and maximal tower top displacement computed for various impact energies are presented in Table 3.

Moreover, maximal initial pressure $\bar{p}_{0}^{\max }$ was introduced because of excessive stresses arising in walls of inflatable structure and its excessive deformation after too strong initial inflation. It was assumed that maximal deflection of inflatable structure can not exceed $0.6 \mathrm{~m}$ and maximal stress after inflation should be smaller than $30 \mathrm{MPa}$. Corresponding maximal initial pressure $\bar{p}_{0}^{\max }$ equals 4 atm and it is independent on impact energy.

In further simulations the tower protected by the pneumatic structure was subjected to impact of the same energy $(0.64 \mathrm{MJ})$ but of various velocities, cf. Table 4 . The total pressure inside the front chamber was equal $1.8 \mathrm{~atm}$ in all cases. The values of the maximal pressure, displacement of the tower top and maximal stresses in the tower wall were observed. Furthermore, all computations were repeated under assumption of adiabatic conditions. The response of the system depends on the excitation in the following way, cf. [17]:

- initial total pressure for which the whole front AIS chamber is crushed depends approximately linearly on impact energy (and it is lower for adiabatic process) 
Table 3

Response of the structure calculated for various impact energies

\begin{tabular}{cccccccc}
\hline $\begin{array}{c}\text { Mass } \\
\text { [ton] }]\end{array}$ & $\begin{array}{c}\text { Velocity } \\
{[\mathrm{m} / \mathrm{s}]}\end{array}$ & $\begin{array}{c}\text { Energy } \\
{[\mathrm{kJ}]}\end{array}$ & $\begin{array}{c}\text { Impulse } \\
{[\mathrm{t} * \mathrm{~m} / \mathrm{s}]}\end{array}$ & $\begin{array}{c}\overline{\mathbf{p}}_{0}^{\min } \\
{[\mathrm{atm}]}\end{array}$ & $\begin{array}{c}\overline{\mathbf{p}}^{\text {max }} \\
{[\mathrm{atm}]}\end{array}$ & $\begin{array}{c}\text { Stress } \\
{[\mathrm{MPa}]}\end{array}$ & $\begin{array}{c}\text { Displ. } \\
{[\mathrm{m}]}\end{array}$ \\
\hline 42.00 & 6 & 756 & 252 & 1 & 7.09 & 193 & 0.00299 \\
67.00 & 6 & 1206 & 402 & 1.45 & 11.67 & 340 & 0.00481 \\
52.00 & 8 & 1664 & 416 & 1.95 & 15.04 & 470 & 0.00499 \\
66.00 & 8 & 2112 & 528 & 2.50 & 18.11 & 611 & 0.00635 \\
80.00 & 8 & 2560 & 640 & 2.95 & 20.51 & 692 & 0.00770 \\
\hline
\end{tabular}

Table 4

Response of the structure to slow and fast impact of the same energy

\begin{tabular}{ccccccccc}
\hline $\begin{array}{c}\text { Mass } \\
\text { [ton] }\end{array}$ & $\begin{array}{c}\text { Velocity } \\
{[\mathrm{m} / \mathrm{s}]}\end{array}$ & $\begin{array}{c}\text { Energy } \\
{[\mathrm{MJ}]}\end{array}$ & $\begin{array}{c}\text { Impulse } \\
{[\mathrm{t} * \mathrm{~m} / \mathrm{s}]}\end{array}$ & $\begin{array}{c}\overline{\mathbf{p}}_{0} \\
{[\mathrm{~atm}]}\end{array}$ & $\begin{array}{c}\overline{\mathbf{p}}^{\text {max }} \\
{[\mathrm{atm}]}\end{array}$ & $\begin{array}{c}\text { Accel. } \\
{\left[\mathrm{m} / \mathrm{s}^{2}\right]}\end{array}$ & $\begin{array}{c}\text { Stress } \\
{[\mathrm{MPa}]}\end{array}$ & $\begin{array}{c}\text { Displ. } \\
{[\mathrm{m}]}\end{array}$ \\
\hline 20.00 & 8 & 0.64 & 160 & 1.8 & 4.879 & 124.6 & 140.1 & 0.00191 \\
35.56 & 6 & 0.64 & 213.36 & 1.8 & 4.916 & 69.33 & 133.8 & 0.00255 \\
80.00 & 4 & 0.64 & 320 & 1.8 & 4.926 & 31.3 & 135.9 & 0.00379 \\
\hline
\end{tabular}

- for given initial inflation maximal pressure depends nonlinearly on impact energy (and it is higher for adiabatic process)

- for the same impact energy maximal ship acceleration increases proportionally to square of ship velocity and decreases inversely proportionally to ship mass

- when the chambers are long and narrow large deformations and stresses appear after initial inflation in the outer wall

- displacement of the tower top is proportional to the impulse of the ship and it is relatively very small

Drawn conclusions agree with Eq. (10) describing simplified air-spring model. On contrary to above examples, in further sections controlled release of pressure will be executed. The following inflatable systems will be considered:

1. Reference passive case: initial pressure adjusted to the harshest ship impact

(i.e. $p_{0}=p_{0}^{\max }=3 \mathrm{~atm}$ ), no pressure release

2. Semi-active: only initial pressure or both initial pressure and constant in time orifice opening adjusted to particular impact

3. Active: orifice opening actively controlled during impact process

\section{Alleviation of ship response}

\subsection{Minimisation of ship acceleration}

We will consider exemplary impact of 60 ton ship approaching the tower with the velocity $6 \mathrm{~m} / \mathrm{s}$, two semi-active and two active schemes of control. In all further examples the inflation of the chambers will be executed during initial $200 \mathrm{~ms}$ and the ship will impact the structure directly afterwards. The distribution of pressures in the cavities will be fixed (100\% in front cavity, $50 \%$ in adjacent cavities, $10 \%$ in other ones) and it will not be subjected to optimisation.

The first purpose of the pressure adjustment is to decrease ship acceleration. In considered $2 \mathrm{D}$ model the equivalence of acceleration and pressure minimisation problems is disrupted due to influence of initial change of chambers volume, forces arising during rubber deformation and change of contact area between ship and inflatable structure during collision. In the simplest semi-active case only initial pressure is adjusted so after inflation mass of the gas inside chambers remains constant. Maximal ship acceleration is computed for each value of initial pressure by executing finite element analysis. Optimal initial pressure $p_{0}^{\text {opt }}$ is searched within the set of allowable initial pressures ranging from $p_{0}^{\min }$ to $p_{0}^{\max }$ by means of gradient method. Minimal ship acceleration of $58.84 \mathrm{~m} / \mathrm{s}^{2}$ (cf. Fig. 7, continuous line) is achieved for the highest admissible initial pressure, which is the consequence of significant expansion of the front chamber after inflation. Thus, semi-active system without pressure release is 

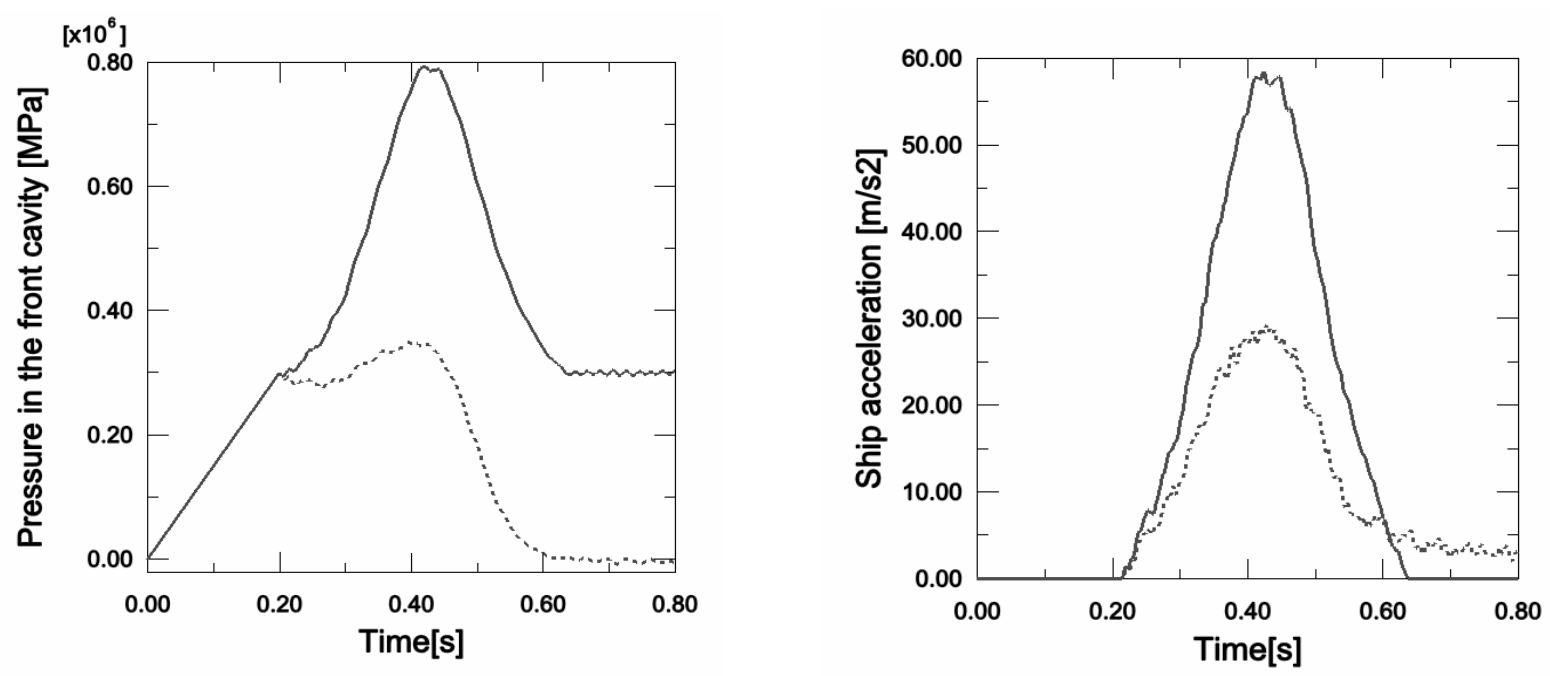

Fig. 7. Passive response (continuous line) and semi-active adaptation with pressure release (dashed line): a) pressure in the front chamber, b) corresponding ship acceleration.

equivalent to the passive one. Let us note that minimal ship acceleration is obtained for the case when only part of the inflatable structure stroke is used which gives the possibilities of further system improvement.

More sophisticated type of semi-active system includes the exhaust valves which are opened at time instant when the ship approaches the inflatable structure. The opening of the valves can be adjusted for particular impact scheme, but it remains constant during whole event. In considered case the air is released from three front chambers which are intrinsic for impact absorption. Similarly as in Section 5.3, gas flow is described by Eq. (4a) with hydrodynamic coefficient $C_{H}$ equal to zero so mass flow rate is proportional to pressure difference between each chamber and environment. By letting the gas out of the cavities we decrease internal pressure and its maximal value is reduced to $3.49 \mathrm{~atm}$. Thus we diminish global force acting on the ship and simultaneously increase depth of inflatable structure compression. Minimal ship acceleration of $29.14 \mathrm{~m} / \mathrm{s}^{2}$ (cf. Fig. 7, dashed line) is obtained for the case when maximal ship displacement achieves its limit i.e. the ship is stopped just before the tower wall. Computed value of corresponding flow resistance coefficient $C_{V}=275 \frac{\mathrm{kPa} \cdot \mathrm{s}}{\mathrm{kg}}$ and maximal mass flow rate $q=1.27 \frac{\mathrm{kg}}{\mathrm{s}}$ allows to choose proper type of controllable valve.

Further decrease of ship acceleration can be obtained by active control of the orifice diameter during impact. In active control strategy the valve remains closed until ship acceleration achieves the level required to stop the ship using the way remaining to the tower. Therefore the time of valve opening is based on actual ship velocity, acceleration and distance to the tower. Originally, the pressure value will be used as a main control parameter since it influences ship acceleration the most directly. Theoretically, optimal pressure can be calculated from equation of ship motion which takes into account the forces arising during compression of inflatable structure:

$$
M a^{*}(t)+\sum_{n=1 . .3} A_{n}(p, t) p_{n}(t)+P^{A I S}(p, t)=0
$$

where $a^{*}(t)$ is assumed value of acceleration, $A_{n}(p, t)$ is area of contact between the ship and each inflatable chamber and summation is performed over three front chambers. The component $P^{A I S}(p, t)$ indicates the force acting on the ship caused by strongly deformed walls of inflatable structure. The disadvantage of the method is that precise value of force $P^{A I S}$ in terms of pressure and actual ship location is required for optimal pressure calculation. Such difficulty can be overcome by comparing actual and optimal state of the system for each time step of finite element analysis. The procedure is started at time instant when values of pressure and acceleration are optimal. Thus, the subsequent actual and optimal state of the system are close to each other and it can be assumed that the force $P A I S$ and the total contact area $A$ are equal for both of them. We obtain simple expression defining pressure modification which has to be applied at each time step: 


$$
\Delta p(t)=\frac{M\left(a^{*}-a(t)\right)}{A(p, t)}
$$

Finally, required mass flow rate and optimal flow coefficient can be calculated by using optimal pressure and chamber volume obtained from finite element analysis.

An alternative numerical approach utilizes flow resistance coefficient, instead of pressure, as a control variable. In this strategy the flow coefficient is proportionally adjusted on several time intervals to achieve ship acceleration possibly close to desired level. The advantage of the method is the possibility of imposing constraints on maximal orifice area. Another effective approach is using the on/off strategy for the valve opening, however in such case much faster operating valve is required.

Described methods of control were implemented in ABAQUS/Standard code by using FORTRAN subroutines. The URDFIL subroutine was utilized to read the actual value of ship acceleration during finite element analysis. The DISP subroutine was used to change actual value of pressure or, alternatively, the UFIELD subroutine was used to change the flow resistance coefficient. The adaptation procedure was started 110 milliseconds after the contact between ship and inflatable structure occurs, when ship acceleration equals $20.7 \mathrm{~m} / \mathrm{s}^{2}$, cf. Fig. 8 (continuous line). After activation of control optimal value of pressure declines gradually for several dozen milliseconds and then it remains at similar level. The ship acceleration is not exactly constant due to vibrations of inflatable structure walls and inaccuracy of numerical scheme of finite element solution. Maximal acceleration obtained by this method is eventually reduced to $21.56 \mathrm{~m} / \mathrm{s}^{2}$, but high mass flow rate of $q=3.9 \frac{\mathrm{kg}}{\mathrm{s}}$ is required at initial stage of impact.

Finally, an attempt to keep constant ship acceleration during the whole time of impact was done. Disadvantageous slow increase of acceleration in passive stage of impact (200-310 ms) can be avoided by additional inflation of the main chamber after the ship approaches inflatable structure (when it is located around $1.1 \mathrm{~m}$ from the tower wall). The pressure has to be increased to $6.6 \mathrm{~atm}$ and as a result sudden growth of acceleration is obtained (cf. Fig. 8, dashed line). Due to the fact that the way of braking the ship is longer than in previous case, required level of acceleration is lower and theoretically equals $a^{*}=16.4 \mathrm{~m} / \mathrm{s}^{2}$. However, after fast additional inflation whole pneumatic structure is strongly vibrating and precise pressure adjustment is difficult. The ship acceleration was finally reduced to $18.76 \mathrm{~m} / \mathrm{s}^{2}$ which constitutes $32 \%$ of ship acceleration in pasive case.

\subsection{Minimisation of ship rebound}

Considered torus-shaped Adaptive Inflatable Structure serves as a docking facility and ship rebound is not a desired phenomenon. Thus, the next control objective is to reduce ship rebound i.e. to minimise ship velocity after collision, possibly to zero. Energy balance for ship collision with inflatable torus is analogous to Eq. (10b) but complemented with the term indicating strain energy of AIS walls. For the case of adiabatic change of gas it reads:

$$
-\Delta E_{k}^{s h i p}+\Delta W^{e x t}=\Delta U+\Delta E^{A I S}+\Delta E^{\text {tower }}-\Delta H
$$

In passive or semi-active system with no pressure release main part of ship kinetic energy $\left(\Delta E_{k}^{s h i p}\right)$ is converted into internal gas energy $(\Delta U)$ and small part is converted into AIS walls strain energy $\left(\Delta E^{A I S}\right)$. Kinetic and potential energy of the tower $\left(\Delta E^{\text {tower }}\right)$ can be neglected due to small tower displacement and velocity. During the rebound stage of impact the gas expands, its internal energy decreases and it is transferred back to the ship. As a result the final kinetic energy of the ship is only $2 \%$ lower than the initial one, cf. Fig. 9.

In contrast to above case, in semi-active or active system with pressure release internal gas energy is not accumulated but it is dissipated by letting the air out of the cavities $(\Delta H)$. Rebound of the ship is related to exergy of the gas (its ability to perform useful work) at time instant when the ship is stopped. Exergy of the gas depends on gas pressure and volume so it can be effectively reduced by applying controlled pressure release.

Let us initially consider semi-active adaptation to impact. Adjustment of initial pressure does not affect the final ship velocity since it does not cause energy dissipation and it will be set to $p_{0}^{\max }=3 \mathrm{~atm}$. Ship rebound can be effectively reduced by semi-active system comprising the valve with constant opening. Minimal final ship velocity of $1.25 \mathrm{~m} / \mathrm{s}$ (cf. Fig. 9b) is obtained in case when whole stroke of inflatable structure is used. Optimal flow resistance coefficient equals $C_{V}=275 \frac{\mathrm{kPa} \cdot \mathrm{s}}{\mathrm{kg}}$, as in case of semi-active acceleration mitigation. Corresponding change of gas mass and gas pressure are presented in Figs 9a and 7a, respectively. In considered example the final gas pressure is 

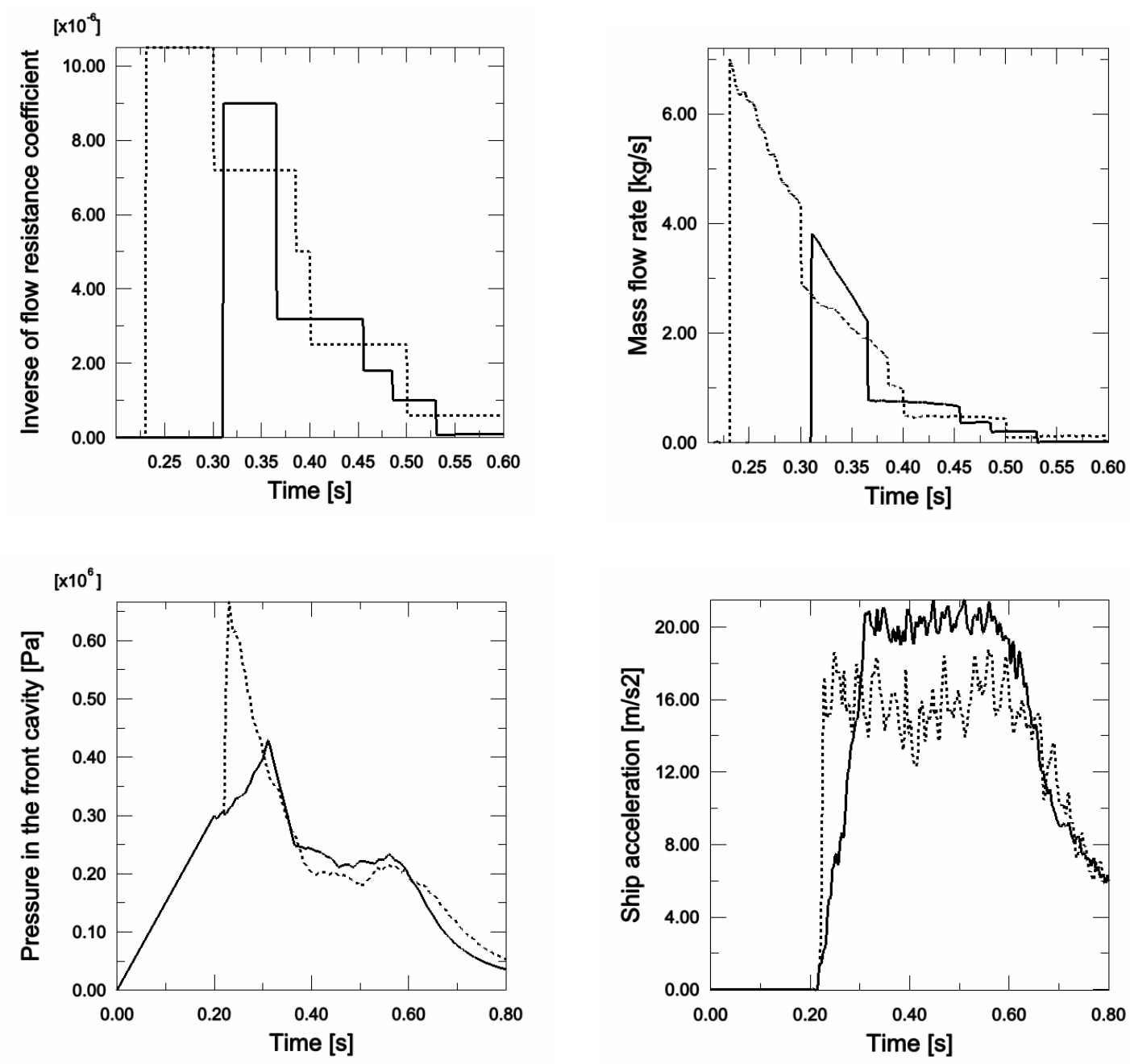

Fig. 8. Two strategies of active acceleration mitigation: a) pressure only released during impact (continuous line), b) additional inflation at the beginning of impact (dashed line).

not completely reduced, but final chamber volume is minimal and work that can be done by gas is relatively small. Therefore, semi-active system is very efficient and allows to dissipate almost $96 \%$ of initial ship energy.

In active strategy the valve opening remains constant while ship is approaching the tower, as in semi-active approach. When ship velocity decreases to zero (at time $0.54 \mathrm{~s}$ ), the valves are fully opened to release surplus of pressure, cf. Fig. 10a (continuous line). This way gas pressure is reduced to atmospheric pressure, gas has no capability of expansion and can not perform useful work. Then, the valve is closed and backward deformation of the chambers is reduced due to arising under-pressure. In this case the URDFIL subroutine was used to read the actual velocity of the ship during simulation. The UFIELD subroutine was utilized to define the valve opening in terms of ship velocity. The result of this strategy is reduction of rebound velocity to $1.05 \mathrm{~m} / \mathrm{s}$ (cf. Fig. 10b). Non-zero final ship velocity is the consequence of ship interaction with strongly deformed pneumatic structure walls which repel the ship.

The influence of inflatable structure walls can be further decreased by minimising their deformation and thereby the amount of strain energy accumulated. In present control strategy the valves remain closed during whole compression stage of impact to achieve the highest possible stiffness of pneumatic torus. The ship is stopped after crushing only part of inflatable structure at time $0.42 \mathrm{~s}$ and then immediate pressure release is executed, cf. Fig. 10a (dashed line). 

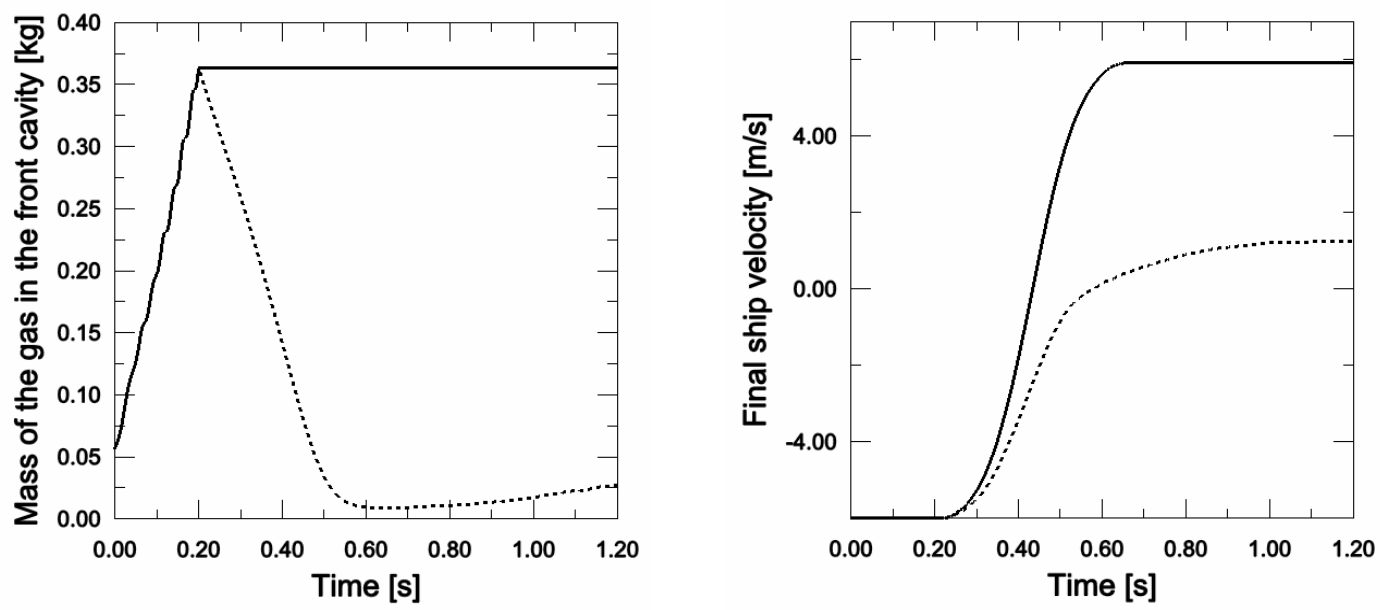

Fig. 9. Passive case (continuous line) and semi-active adaptation with pressure release (dashed line): a) mass of the gas in the front cavity, b) corresponding velocity of the ship.
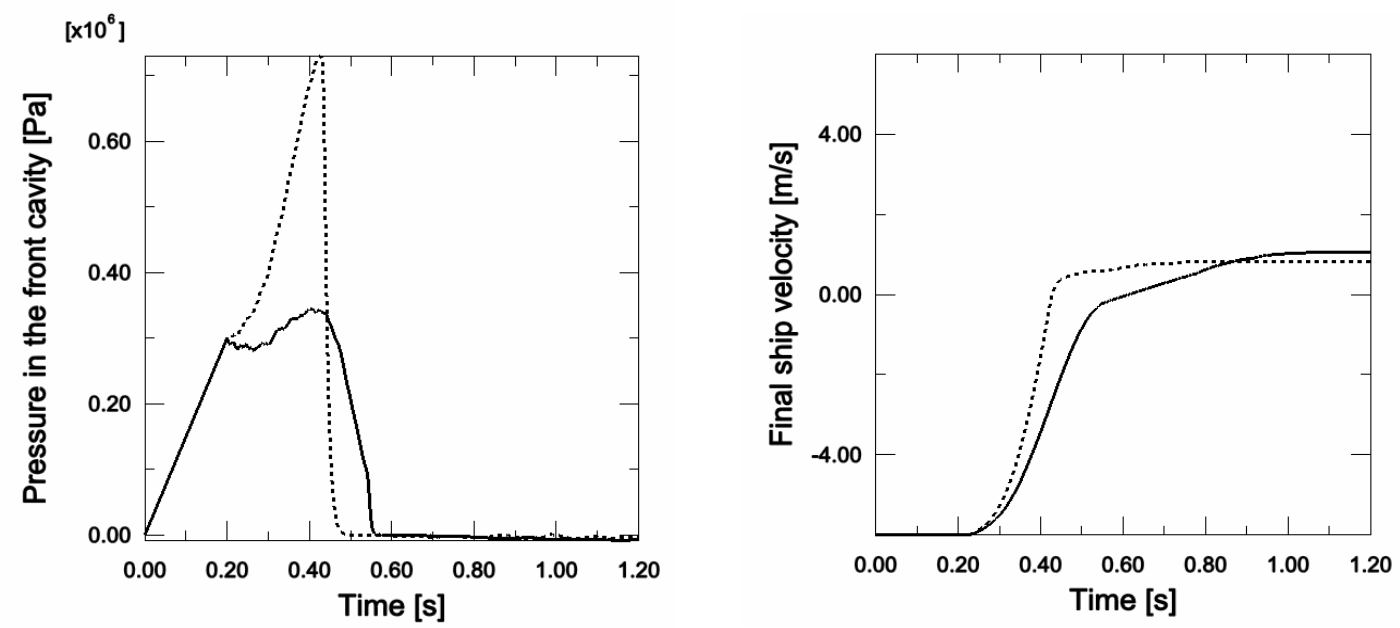

Fig. 10. Active mitigation of ship rebound: a) pressure of the gas in the cavity, b)corresponding final velocity of the ship.

Finally, the rebound velocity is reduced to $0.82 \mathrm{~m} / \mathrm{s}$ which means that more than $98 \%$ of the initial ship energy is dissipated.

Additional possibility of rebound mitigation is changing the stiffness of diaphragms between front AIS chambers. This strategy helps to reduce significantly high strain energy accumulated in deformed outer AIS wall and transfer it into kinetic energy of other parts of pneumatic structure. However, such method is rather theoretical and seems to be difficult to apply in practise.

\section{Mitigation of the tower response}

\subsection{Minimisation of stresses in tower wall}

Another purpose of applying the inflatable structure is mitigation of the tower response to impact. In particular, pneumatic structure reduces local stresses in the front tower wall by preventing direct contact of the ship and the 

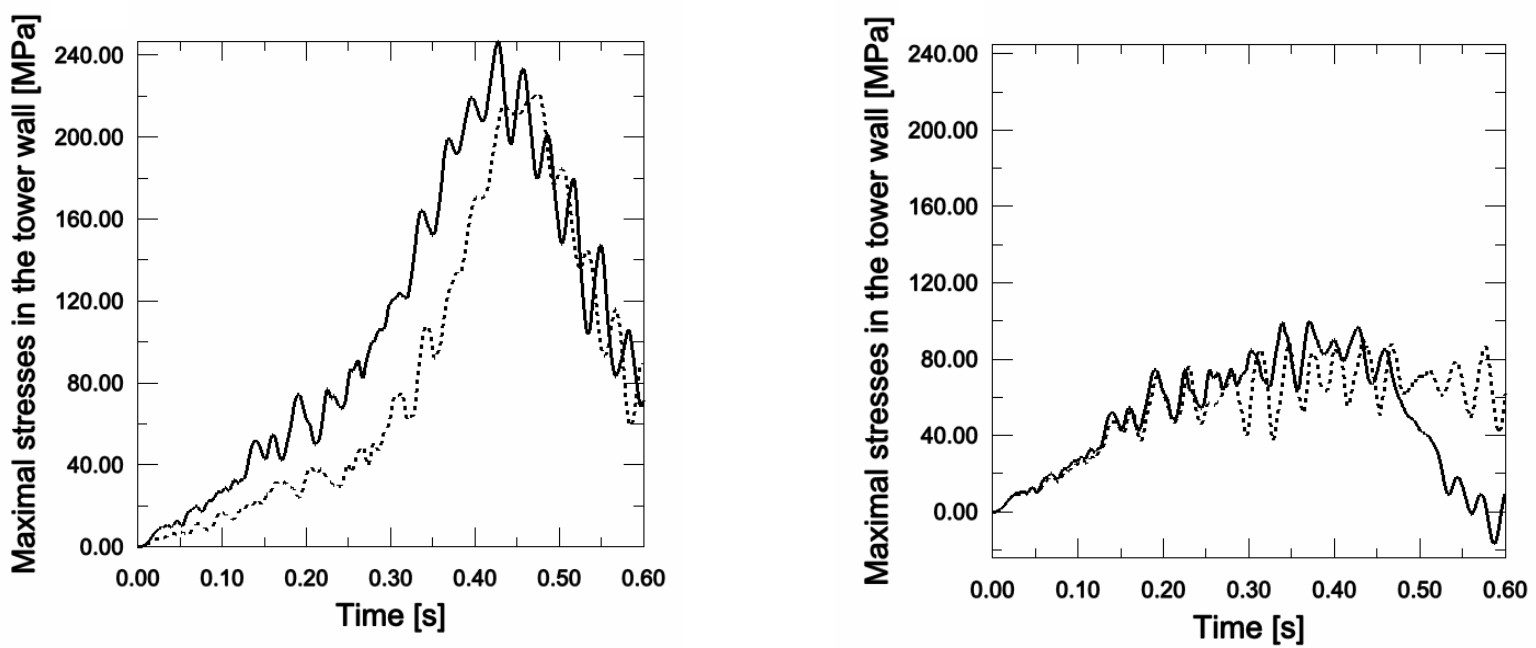

Fig. 11. Stress in tower wall: a) Passive system (continuous line) and semi-active system without pressure release (dashed line), b) Semi-active (continuous line) and active (dashed line) control with pressure release.

tower, which is achieved by applying initial pressure higher than $p_{0}^{\min }$ for each impact. Front tower wall is subjected to bending caused by pressure loading and level of its stress depends approximately on actual value of pressure. Therefore, the minimization of stresses is equivalent to minimization of front chamber pressure.

In semi-active system without pressure release tensile stresses can be reduced to $221.9 \mathrm{MPa}$ (cf. Fig. 11a) by applying initial pressure considerably lower than in passive case $\left(p_{0}=1.7 \mathrm{~atm}\right)$. The semi-active system with pressure release is the most efficient when maximal initial pressure $p_{0}^{\max }$ and whole stroke of inflatable structure are utilized, cf. Figs 7a and 9a for pressure and mass plots, respectively. In such system maximal pressure is decreased to $3.49 \mathrm{~atm}$ and stresses in tower wall are diminished to $104.5 \mathrm{MPa}$ (cf. Fig. 11b, continuous line).

In fully active system, pressure is adjusted to constant, possibly lowest level in order to minimise stresses in tower wall. For impact energies lower than 1.25 MJ such pressure does not exceed maximal initial pressure and hence it may remain constant during the whole process. For impact energy higher than 1.25 MJ optimal strategy assumes initial stage of pressure increase and activation of control when pressure achieves level which enables stopping the ship in the vicinity of tower wall.

For considered impact of 60 tons ship with the velocity $6 \mathrm{~m} / \mathrm{s}$ optimal pressure $p^{o p t}=2.68$ atm was found by performing multiple finite element analysis. Actual mass of the gas within chambers was calculated by using actual chambers volumes obtained from the numerical simulation and ideal gas law. The mass of the gas in front cavity increases during the inflation stage and it is reduced strongly to keep pressure on constant level when the ship crushes the chamber. During the rebound stage of impact mass of the gas increases back to initial level, cf. Fig. 12a. The mass flow rate through the valve is given as the mass derivative over time:

$$
q(t)=\frac{d m}{d t}=\frac{\left(p^{o p t}+p_{A}\right)}{R T_{0}} \cdot \frac{d V(t)}{d t}
$$

Finally, the flow resistance coefficient that provides appropriate mass exchange under given conditions of pressure difference can be calculated using Eq. (4a). During the impact time pressure remains constant so the inverse of flow resistance coefficient is proportional to mass flow rate $q(t)$.

Active system with the flow resistance coefficient adjustment presented in Fig. 12b provides significant reduction of pressure in comparison to semi-active case. However, high frequency vibrations of the tower wall arise and aggravate significant minimisation of stresses. Finally, the stresses are reduced to $91.9 \mathrm{MPa}$ and active system is $12 \%$ more effective than the semi-active one. 

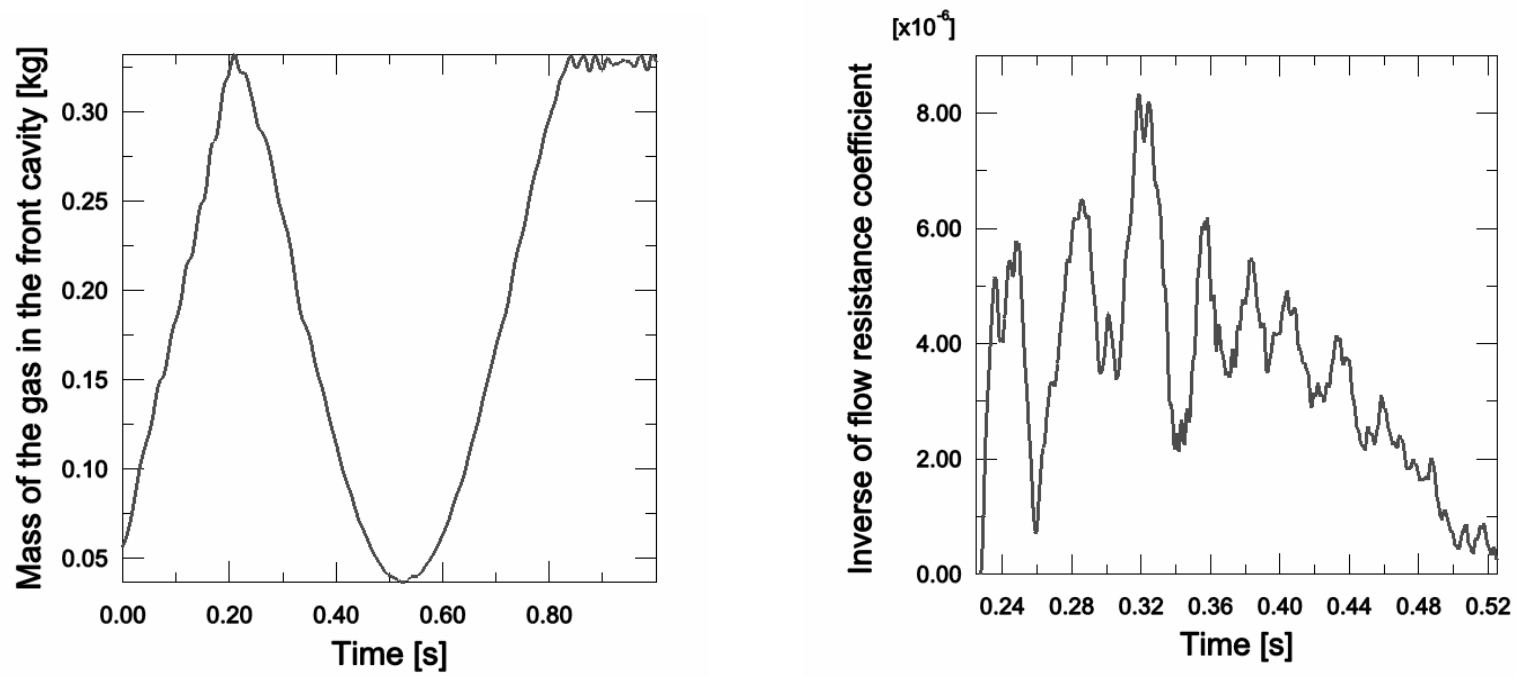

Fig. 12. Active strategy of stress minimisation: a) mass of the gas in the cavity, b) inverse of flow resistance coefficient during compression stage of impact.

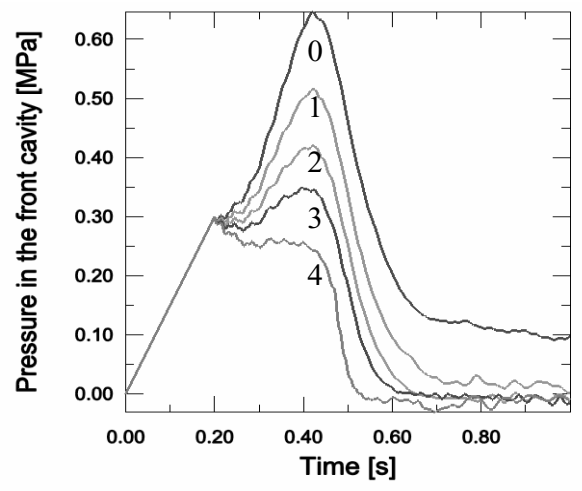

\begin{tabular}{|c|c|c|c|}
\hline No. & $\begin{array}{c}\mathrm{Cv} \\
{\left[\mathrm{Pa}^{*} \mathrm{~s} / \mathrm{kg}\right]}\end{array}$ & $\begin{array}{l}\text { Max press. } \\
\text { [atm] }\end{array}$ & Displ. [m] \\
\hline 0 & 1000000 & 6.46 & 0.00405 \\
\hline 1 & $500 \quad 000$ & 5.15 & 0.00348 \\
\hline 2 & $350 \quad 000$ & 4.19 & 0.00299 \\
\hline 3 & 275000 & 3.49 & 0.00267 \\
\hline 4 & $200 \quad 000$ & 3.00 & 0.00336 \\
\hline
\end{tabular}

Fig. 13. Semi-active mitigation of tower vibrations: a) change of pressure and b) corresponding response of the tower.

\subsection{Mitigation of tower vibrations}

The last goal of pressure adjustment is to minimise the amplitude of tower vibrations after impact. This objective can be alternatively understood as a minimization of the energy transmitted to the tower during ship collision. Due to the fact that impact time is relatively short in comparison to the period of tower vibration, maximal tower displacement depends on ship impulse, cf. [13]. Impulse transmitted to the tower is proportional to mass of the ship and difference of its initial and final velocity. Hence, minimisation of tower vibrations is approximately equivalent to minimisation of ship rebound and vibration amplitude can be reduced maximally by $50 \%$.

In semi-active system without pressure release maximal tower displacement is almost independent on initial pressure and equals $4.58-4.60 \mathrm{~mm}$, which agrees with the result obtained by using simple model of elastic collision $(4.68 \mathrm{~mm})$. By using semi-active system with pressure release, amplitude of tower vibration is reduced as presented in Fig. 13. The best result $(2.67 \mathrm{~mm})$ is obtained for the highest flow resistance coefficient for which the ship does not hit the tower wall.

Active strategies applied for minimisation of ship rebound were also investigated. The most effective adaptation procedure assumes constant valve opening as in semi-active approach and additional release of pressure at time 
instant when ship velocity approaches zero. By using such strategy maximal tower displacement is reduced to $2.51 \mathrm{~mm}$. Keeping the valve closed and executing pressure release only when the ship is stopped (which is the most effective for rebound mitigation) gives slightly larger tower displacement, probably due to shorter impact time. Let us note that the equivalence of minimisation of ship rebound and tower vibrations holds only when structure own frequency is adequately low. For structures with short period of vibrations another control strategy has to be applied.

\section{Conclusions and future steps}

The proposed Adaptive Inflatable Structure surrounding the tower can effectively protect the offshore wind turbine and the ship in case of collision. Adjustment of initial pressure and controlling its release adapts the inflatable structure to various impact conditions and significantly increases the system effectiveness. A controlled release of pressure helps to dissipate a major part of the impact energy and thus to decrease ship rebound and tower vibrations. Precise control of the valve flow enables minimisation of ship acceleration and reduction of stress in the tower wall.

Semi-active control is the most efficient when maximal admissible initial pressure is applied and flow resistance coefficient is adjusted to utilize the whole stroke of pneumatic structure. Conducted simulations clearly indicate the superiority of semi-active structure over the passive one since it reduces ship acceleration and stresses in tower wall by over $50 \%$ and ship rebound velocity by nearly $80 \%$. Active pressure control was found to be more effective for mitigation of ship response (profit up to $36 \%$ in comparison to semi-active case) than for alleviation of tower vibration and stresses (profit up to 12\%). However, the execution of active control schemes requires more sophisticated electronic control system and more power supply.

In the following stages of research the possibilities of constructing high speed and stroke controllable valve should be examined. Furthermore, the description of the flow should be adjusted to experimental data. During the design process of particular inflatable structure full 3D model should be used for selected numerical computations due to limitations of simplified modelling described in Section 5.4. Especially, more accurate modelling of the material chosen for inflatable torus (for instance reinforced rubber) is expected to be required. Finally, an experimental verification of the whole system is necessary to test out its functioning and effectiveness.

\section{Acknowledgement}

The authors would like to gratefully acknowledge the financial support through the Polish research project MATINT, PBZ-KBN-115/T08/2004 (2005-2008) “Intelligent materials made of metals, ceramics and polymers: design, production, properties and applications". The part of the work was done during first author visit at VTT Technical Research Centre of Finland. The author would like to gratefully acknowledge the support of VTT in accomplishing this work. Essential help provided by Jaakko Heinonen and Tuomo Kärnä is especially highly appreciated.

Part of the article will be published as chapter of the book "Smart Technologies for Safety Engineering", cf. [6].

\section{References}

[1] J.F. Wilson, Dynamics of Offshore Structures, Wiley, New York, USA, 1984

[2] T. Kärnä and K. Kolari, Mitigation of dynamic ice actions on offshore wind turbines, Third European conference on Structural Control, 12-15 July 2004 Vienna.

[3] A. Mróz and T. Kärnä, Mitigation of ice loading. Feasibility study of semi-active solution, VTT Working Papers 39 (2005).

[4] F. Biehl and E. Lehmann, Collisions of ships and offshore wind turbines: Calculation and risk evaluation, Proceedings of the International Conference on Offshore Mechanics and Arctic Engineering OMAE, 2006.

[5] F. Biehl, Collision Safety Analysis of Offshore Wind Turbines, LS-DYNA Forum, Bamberg, 2005

[6] J. Holnicki-Szulc, ed., Smart Technologies for Safety Engineering, J.Wiley, 2008, in preparation.

[7] J. Holnicki, G. Mikułowski, A. Mróz and P. Pawłowski, Adaptive Impact Absorption, The Fifth International Conference on Engineering Computational Technology, September 2006, Las Palmas de Gran Canaria, Spain.

[8] EU Project ADLAND IST-FP6-2002-2006, http://smart.ippt.gov.pl/adland/.

[9] P. Pawłowski, ADLAND Deliverable 9: Hardware Controller. 
[10] K. Sekula and J. Holnicki-Szulc, Comparison of real time impact load identification procedures, III ECCOMAS thematic conference on smart structures and materials, July 9-11, 2007, Gdansk, Poland.

[11] I. Yeh, L. Chai and N. Saha, Application of ALE to airbag deployment simulation, International Journal of Vehicle Safety 1(4) (2006), $348-365$.

[12] G.J. Van Wylen and R.E. Sonntag, Fundamentals of Classical Thermodynamics, John Wiley \& Sons, 1978.

[13] R.W. Clough and J. Penzien, Dynamics of Structures, McGraw-Hill, 1975.

[14] ABAQUS ver. 6.52005 User's Manual. ABAQUS Inc. Rising Sun Mills, USA.

[15] T. Belytschko, W.K. Liu and B. Moran, Nonlinear Finite Elements for Continua and Structures, Wiley, 2000.

[16] R.D. Cook, D.S. Malkus and M.E. Plesha, Concepts and Applications of Finite Element Analysis, John Wiley \& Sons, New York, 1989.

[17] C. Graczykowski and J. Heinonen, Adaptive Inflatable Structures for protecting off-shore wind turbines against ship collision, VTT Working Papers 59 (2006). 

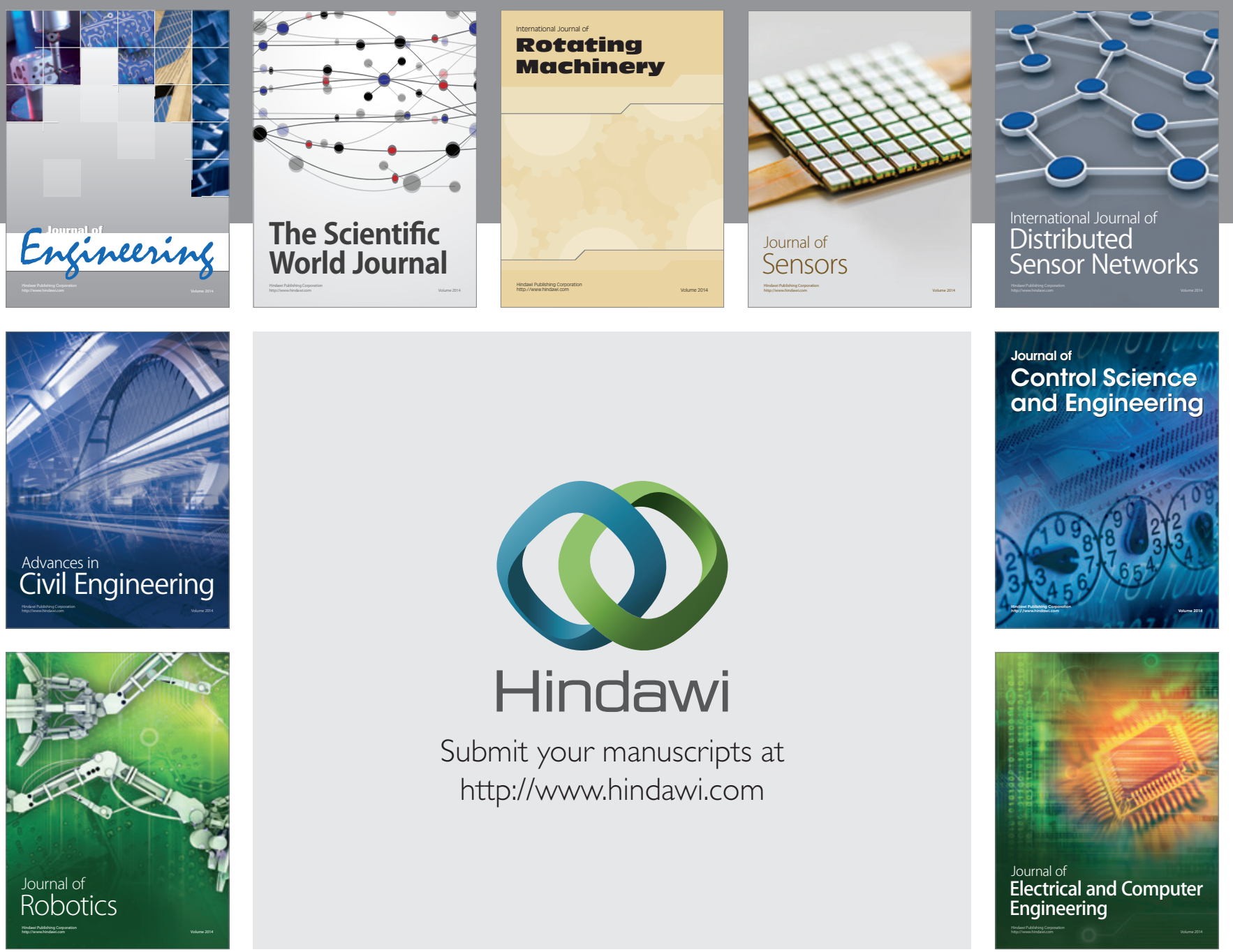

Submit your manuscripts at

http://www.hindawi.com
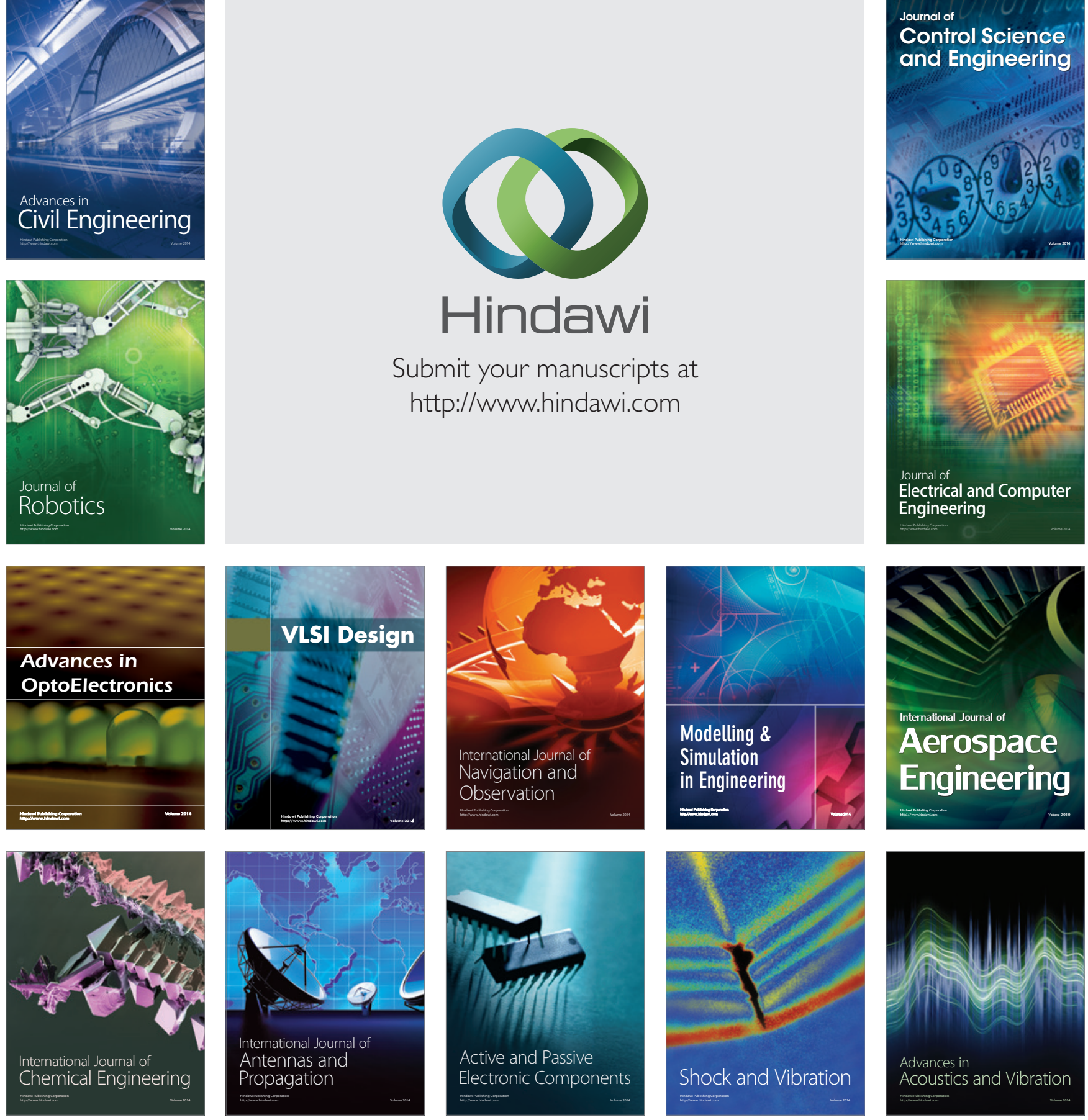